\title{
Association of a BTLA gene polymorphism with the risk of rheumatoid arthritis
}

\author{
Shih-Chang $\operatorname{Lin}^{1,2,3, *}$, Chia-Chen Kuo ${ }^{1}$ \& Chia-Hsi Chan ${ }^{1}$ \\ ${ }^{1}$ Division of Allergy and Immunology, Department of Internal Medicine, Cathay General Hospital, 280 Jen-Ai \\ Rd Section 4, Taipei, Taiwan; ${ }^{2}$ The Laboratory of Allergy and Immunology, Cathay Medical Research \\ Institute, Hsichih, Taipei Shien, Taiwan; ${ }^{3}$ Department of Medicine, School of Medicine, Fu Jen Catholic \\ University, Hsinchuang, Taipei Shien, Taiwan
}

Received 12 April 2006; accepted in revised form 28 August 2006

(C) 2006 National Science Council, Taipei

Key words: B and $\mathrm{T}$ lymphocyte attenuator (BTLA), rheumatoid arthritis (RA), single nucleotide polymorphism (SNP)

\begin{abstract}
Summary
$\mathrm{B}$ and $\mathrm{T}$ lymphocyte attenuator (BTLA) is an immuno-inhibitory receptor with the ability to deliver inhibitory signal for suppressing lymphocyte activation. To test the potential association of the human BTLA gene with the development of rheumatoid arthritis (RA), a genetic case-control association study was conducted, by using a single nucleotide polymorphism (SNP), C+800T SNP, in the exon 5 of the human BTLA gene for genotyping 93 RA patients and 294 normal control individuals. The results showed that there is statistically significant difference in the genotype distributions between RA and control groups $(p=0.022)$. When compared with the heterozygous genotype ( $\mathrm{C} / \mathrm{T}$ genotype), the homozygous genotype $(\mathrm{C} / \mathrm{C}$ or $\mathrm{T} / \mathrm{T}$ genotype) appears to confer the increased risk of the RA susceptibility with the odds ratio of $1.88(p=0.015)$. These data indicate the significant association between the C+800T SNP in the BTLA gene with the RA susceptibility.
\end{abstract}

\section{Introduction}

Lymphocyte activation is regulated by stimulatory and inhibitory signals delivered through the interaction between stimulatory and inhibitory receptors with their cognate ligands. Many of these receptors and ligands belong to the CD28 and $\mathrm{B} 7$ families (see recent reviews in references [1] and [2]). Among these receptors, CD28 and ICOS are well known to deliver stimulatory signal for $\mathrm{T}$ cell activation, whereas CTLA-4 (CD152) functions as an inhibitory receptor to counteract the CD28 action in T cells. Recently, two additional inhibitory receptors, programmed

*To whom correspondence should be addressed. Fax: + 886-227082121-ext-3211; E-mail: sclin@cgh.org.tw death-1 (PD-1) and B and T lymphocyte attenuator (BTLA) have been shown to deliver inhibitory signal to down-regulate the activation of both $\mathrm{T}$ and $\mathrm{B}$ cells [3-5]. It appears that the balance between stimulatory and inhibitory signals in lymphocytes may play an important role in regulating normal immune responses, but preventing the development of autoimmune diseases, as evidenced by the immunodeficiency phenotypes in mice deficient in CD28 or ICOS and the phenotypes of autoimmunity and immune hyperactivity in mice deficient in CTLA-4, PD- 1 or BTLA gene [4, 6-10].

BTLA is a CD28-family member expressed in T and B lymphocytes, and its expression in lymphocytes is increased after cell activation [4]. In $T$ cells, it appears that only Th1 cells, but not 
Th2 cells, express BTLA after T cell differentiation, indicating the important role of BTLA in regulating the Th1-mediated immune responses. BTLA can negatively regulate immune responses as evidenced by the finding that BTLA deficiency in mice results in enhanced $\mathrm{T}$ cell proliferation and specific antibody responses and the increased susceptibility to experimental autoimmune encephalomyelitis. The inhibitory signal of BTLA appears to be mediated by the recruitment of SHP-1 and SHP-2 to two immuno-receptor tyrosine inhibitory motifs (ITIMs) in the cytoplasmic region of BTLA $[4,5]$. The ligand for BTLA was recently identified to be herpesvirus entry mediator (HVEM), a member of the TNFR family $[11,12]$. HVEM-deficient mice showed enhanced $\mathrm{T}$ cell proliferation and CD4 $+\mathrm{T}$ cell-dependent proinflammatory cytokine production in response to concanavalin A stimulation, and, similar to the BTLA-deficient mice, they were also found more susceptible to experimental autoimmune encephalomyelitis [13]. These findings suggest the important role of the BTLA-HVEM interaction in suppressing the immune responses and autoimmunity. Thus, PD-1 and BTLA appears to govern the peripheral tolerance according to the wide expression of PD-1 ligands and HVEM in different tissues [14].

Rheumatoid arthritis (RA) is an autoimmune disease, characterized by the synovitis with joint destruction. Although the pathogenesis of RA remains unclear, the synovitis in RA patients appears to be mainly mediated by Th1 cells [1517]. Due to the biased expression of BTLA in Th1 cell, but not Th2 cells, after $\mathrm{T}$ cell activation and the immuno-inhibitory function of the BTLAHVEM interaction, it is possible that BTLA participates in the control of RA development. Therefore, it is hypothesized that BTLA gene is associated with the RA susceptibility. To test this hypothesis, the presence of a single nucleotide polymorphism (SNP), C+800T, located in the exon 5 of the human BTLA gene, was confirmed in the Taiwanese population, as shown in Figure 1. This nucleotide variation leads to the alteration in the encoded amino acid residue near the proximal ITIM motif of the cytoplasmic region of BTLA. By using this SNP for genotyping, a genetic case-control association study was then conducted to test the potential association of BTLA with the RA susceptibility in the Taiwanese population.

\section{Patients and methods}

\section{Study populations}

Total 93 RA patients were recruited into this study from Cathay General Hospital, Taipei, Taiwan. The classification criteria of American College of Rheumatology were used for the diagnosis of RA [18]. 294 healthy blood donors without the family history of any autoimmune disease were recruited into the control group. The demographic characteristics of normal control and RA groups are shown in Table 1. All patients and control individuals recruited in this study are Taiwanese. The study was approved by the institutional review board for research ethics, and all blood donors provided informed consent.

\section{Genomic DNA extraction}

Genomic DNA for each RA patient and control individual was extracted from peripheral blood leukocytes by using the QIAamp DNA Blood Mini Kit (Qiagen) according to the manufacturer's protocol.

\section{Genotyping by nested polymerase chain reaction $(P C R)$ and restriction analysis}

The genomic region surrounding the $\mathrm{C}+800 \mathrm{~T}$ SNP (rs9288952 in NCBI SNP database) in the exon 5 of human BTLA gene was amplified by nested PCR, using purified genomic DNA and specific outer and inner primer sets. The first round PCR was conducted for a 30 -cycle amplification $\left(1 \mathrm{~min}\right.$ at $94{ }^{\circ} \mathrm{C}, 1 \mathrm{~min}$ at $55^{\circ} \mathrm{C}$ and $1 \mathrm{~min}$ at $72{ }^{\circ} \mathrm{C}$ ) by using $100 \mathrm{ng}$ of genomic DNA as the template and 100 pmole of each outer primer in a PCR buffer containing $2.5 \mathrm{mM}$ dNTP, $2.5 \mathrm{mM}$ $\mathrm{MgCl}_{2}$ and 1 unit Taq polymerase (Boehringer Mannheim). The sequences of outer primers used in the first round PCR are 5'-AGAAGCAAGCACCAGGCAAAA-3' and 5'-GACCCAAGCACTAACATGAACA- $3^{\prime}$. The second round PCR was then conducted for a 40-cycle amplification $\left(1 \mathrm{~min}\right.$ at $94{ }^{\circ} \mathrm{C}, 1 \mathrm{~min}$ at $60^{\circ} \mathrm{C}$ and $1 \mathrm{~min}$ at $72{ }^{\circ} \mathrm{C}$ ) by using $3 \mu \mathrm{l}$ of first round PCR product and 100 pmole of each inner primer in a PCR buffer containing $2.5 \mathrm{mM}$ dNTP, $2.0 \mathrm{mM} \mathrm{MgCl} 2$ and 0.5 unit Taq polymerase. Two different top-strand inner primers were used in the second 


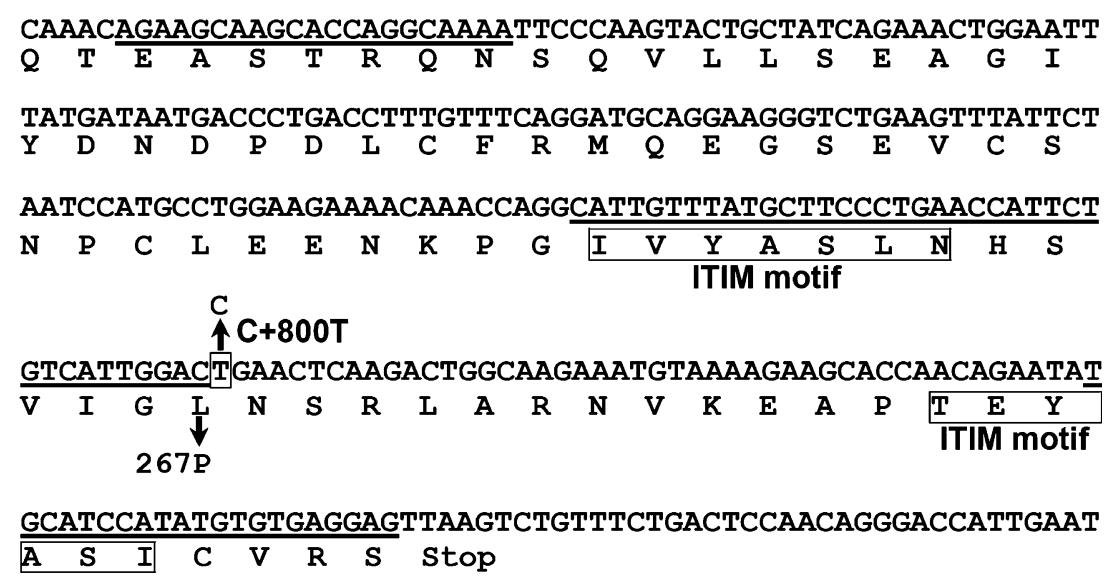

\section{GATCAGCATGTTGACATCATTGTCTGGGCTCAACAGGATGTCAAATAATATTTCTCA}

ATTTGAGAATTTTTACTTTAGAAATGTTCATGTTAGTGCTTGGGTCTTAAGGGTCCA

Figure 1. The partial sequence of the exon 5 of the human BTLA gene and the encoded amino acid sequence. The nucleotides are numbered from the first base of the published BTLA cDNA sequence (Genbank\# NM_181780) [4], and the C+800T SNP is indicated. The corresponding sequences for designing nested PCR primers are underlined. The boxed amino acid sequences are two ITIM motifs in the intracellular domain of the BTLA protein.

round PCR for further detection of different alleles of the C+800T SNP by the restriction fragment length polymorphism (RFLP) analysis. One topstrand inner primer, KpnI primer, with the sequence of 5'-CATTGTTTATGCTTCCCTGAACCATTCTGTCATgGtAC-3' contains two mismatched nucleotides (lowercased) introduced to create a $K p n I$ restriction site in the DNA fragment amplified from the $\mathrm{C}$ allele, but not $\mathrm{T}$ allele. Whereas, the other top-strand primer, Alw NI primer, with the sequence of $5^{\prime}$-ATGCTTCCCTGAACCATTCTGTCcagGGAC-3' contains three mismatched nucleotides (lowercased) introduced to create an $A l w$ NI restriction site in the DNA fragment amplified from the T allele, but not $\mathrm{C}$ allele. The KpnI primer and the bottom-strand inner primer with the sequence of
5'-CTCCTCACACATATGGATGCAT-3' were used for genotyping in the second round PCR to amplify a 105-bp DNA fragment, which was digested by KpnI into 36-bp and 69-bp DNA fragments. Then, the AlwNI primer and the same bottom-strand inner primer were used in the second round PCR to repeat the genotyping and the PCR amplified a 97-bp DNA fragment, which was digested by AlwNI into 28-bp and 69bp DNA fragments. The DNA fragments were then visualized with ethidium bromide in $12 \%$ polyacrylamide gels. Different genotypes, $\mathrm{T} / \mathrm{T}$, $\mathrm{T} / \mathrm{C}$ and $\mathrm{C} / \mathrm{C}$, were therefore determined by evaluating the capability of KpnI or AlwNI to digest nested PCR products, as shown in Figure 2. In addition, the presence of $\mathrm{C}+800 \mathrm{~T}$ SNP was also confirmed by the direct sequenc-

Table 1. Demographic characteristics of normal controls and RA patients.

\begin{tabular}{lll}
\hline & Normal controls & RA patients \\
\hline Case numbers & 294 & 93 \\
Female: male (\% female) & $218: 76(74.1 \%)$ & $74: 19(79.6 \%)$ \\
Age (mean \pm standard deviation) & $46.7 \pm 15.2$ & $53.8 \pm 14.5$ \\
Rheumatoid factor (RF) & $\mathrm{ND}^{\mathrm{a}}$ & All recruited patients have positive RF \\
Treatment & $\mathrm{NA}^{\mathrm{b}}$ & All recruited patients require the treatment of methotrexate \\
\hline
\end{tabular}

\footnotetext{
${ }^{a}$ Not done.

b Not applicable.

${ }^{\mathrm{c}}$ Patients with the relatively severe disease activity were selected into this study.
} 


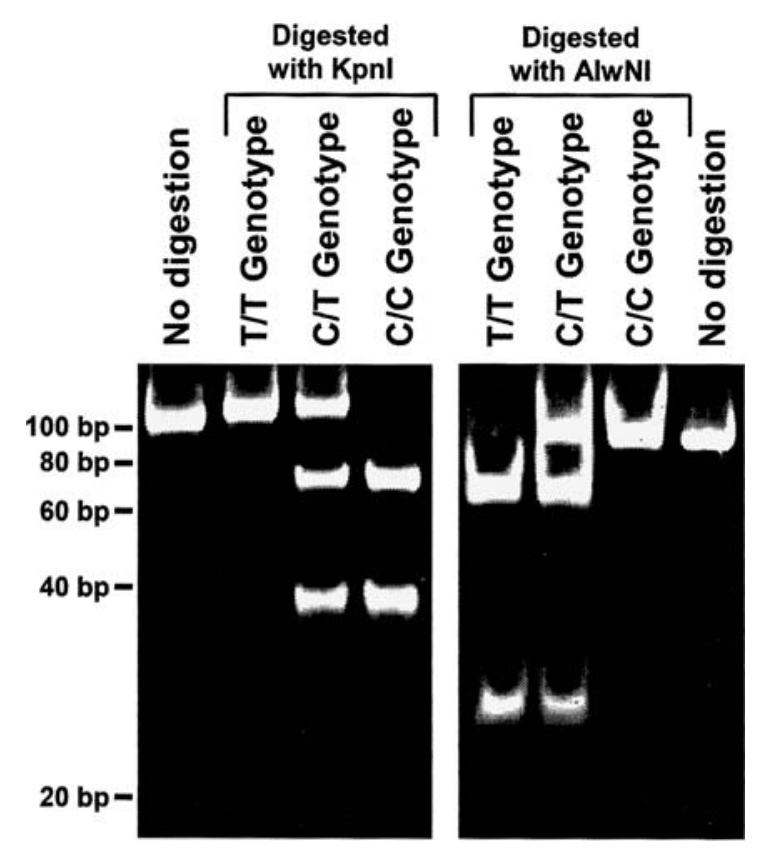

Figure 2. Genotyping by nested PCR with the restriction analysis. Examples of determining the genotypes by the ability of $K p n \mathrm{I}$ or $A l w \mathrm{NI}$ restriction enzyme to digest nested PCR products. The DNA fragments were visualized in polyacrylamide gels stained with ethidium bromide.

ing of some nested PCR products with ABI 377 Genetic Analyzer (Mission Biotech, Taiwan).

\section{Statistical analysis}

The genotype distribution, allele frequencies, allele carriage frequencies (the percentage of individuals carrying at least one copy of $\mathrm{T}$ or $\mathrm{C}$ allele), and homozygous (individuals carrying $\mathrm{C} / \mathrm{C}$ or $\mathrm{T} / \mathrm{T}$ genotype) and heterozygous (individuals carrying $\mathrm{C} / \mathrm{T}$ genotype) genotype frequencies were calculated by direct counting. Chi-square test with two degrees of freedom was used to determine the significance of the difference in genotype distributions of RA and normal groups. Chi-square test with Yates' correction was used to test the significance of differences in $2 \times 2$ contingency tables for allele frequencies and allele carriage frequencies and for homozygous vs heterozygous genotype comparison. Two-sided $p$-values were calculated and considered significant if less than 0.05. An odds ratio (OR) and a Cornfield's 95\% confidence interval (CI) were calculated for a statistically significant result.

\section{Results \\ Genotyping for the C+800T SNP of the human BTLA gene}

An SNP in the exon 5 of the human BTLA gene has been documented in NCBI SNP database (rs9288952). This SNP was named as $\mathrm{C}+800 \mathrm{~T}$ SNP for its position at +800 nucleotide, counting from the first base of the translation start codon in the published cDNA sequence (Genbank\# NM_181780) [4]. The nucleotide substitution from $\mathrm{C}$ to $\mathrm{T}$ leads to the alteration of encoded amino acid residue from leucine to proline in the intracellular domain of BLTA protein at residue 267 (Figure 1). To detect the presence of this SNP in the Taiwanese population, in this study, nested PCR was performed to amplify the genomic region flanking the C+800T SNP, followed by the subsequent restriction fragment length polymorphism (RFLP) analysis. Specific inner primers of nested PCR were designed to create a $K p n I$ restriction enzyme site for the DNA fragment amplified from the $\mathrm{C}$ allele, but not $\mathrm{T}$ allele, and therefore, individuals bearing the $\mathrm{C}$ allele were recognized by the ability of $K p n I$ to digest the nested PCR product in the RFLP analysis, as shown in Figure 2. Then, each individual was genotyped again by using another set of inner primers in nested PCR, which were designed to create an $A l w$ NI restriction enzyme site for the DNA fragment amplified from the $\mathrm{T}$ allele, but not $\mathrm{C}$ allele, and therefore, AlwNI restriction enzyme was used to detect the presence of the $\mathrm{C}$ allele for each individual in the RFLP analysis (Figure 2). After genotyping with the use of $K p n \mathrm{I}$ and $A l w \mathrm{NI}$ restriction enzyme digestion, all individuals in this study were confirmed to contain $\mathrm{C}$ and/or $\mathrm{T}$ allele, indicating the presence of the $\mathrm{C}+800 \mathrm{~T}$ SNP in the Taiwanese population.

\section{Association of the BTLA C+800T SNP with the $R A$ susceptibility}

To determine whether BTLA is associated with the RA susceptibility, the genetic case-control association study was conducted by using this $\mathrm{C}+800 \mathrm{~T}$ SNP in the BTLA gene. Each normal control and RA patient was classified into the $\mathrm{C} / \mathrm{C}, \mathrm{C} / \mathrm{T}$ or $\mathrm{T} / \mathrm{T}$ genotype group. Then, the genotype distribution, 
allele frequency and allele carriage frequency in control and RA groups were calculated and compared by the statistical analysis. It was found that the genotype distribution in the control group is in the Hardy-Weinberg equilibrium, as shown in Table 2. On the contrary, the genotype distribution in the RA group appears to deviate from the Hardy-Weinberg equilibrium and has the statistically significant difference from the genotype distribution in the control group $(p=0.022)$. However, the allele frequencies and the $\mathrm{C}$ and $\mathrm{T}$ allele carriage frequencies between control and RA groups were not significantly different. When each individual was classified into the homozygous genotype group (carrying $\mathrm{C} / \mathrm{C}$ or $\mathrm{T} / \mathrm{T}$ genotype) or the heterozygous group (carrying the $\mathrm{C} / \mathrm{T}$ genotype) for the statistical comparison, it was found that the homozygous genotype frequency in the RA group is different from that in the normal group with the statistical significance $(p=0.015)$ (Table 2). The homozygous genotype appears to confer the increased risk for the RA development with the odds ratio of 1.88 and $95 \%$ confidence interval of 1.12 to 3.17 . The statistical significance in this study is not due to the sampling bias for the RA group, because, when the normal controls and RA patients were also genotyped for an SNP in the human cytokine-inducible SH2-containing protein (CIS) gene, the genotype distribution of that CIS SNP in RA group is in Hardy-Weinberg equilibrium, and is comparable with that in the control group (data not shown). In addition, it may be argued that the association of the BTLA $\mathrm{C}+800 \mathrm{~T}$ SNP with RA susceptibility is not specific to a Th1 cell-mediated autoimmune disease. Therefore, we also tried to determine whether the BTLA C+800T SNP is associated with the susceptibility of systemic lupus erythematosus (SLE), a mainly Th2 cell-mediated autoimmune disease. The BTLA C +800T SNP was then used to genotype 73 SLE patients, and it was found that the genotype distribution in SLE patients was in Hardy-Weinberg equilibrium, similar to the genotype distribution in the control group of this study (data not shown), suggesting the lack of association between the BTLA C+800T SNP and the SLE susceptibility.

\section{Discussion}

$\mathrm{RA}$ is an autoimmune disease with the unknown pathogenesis, while its development appears to be influenced by the complex genetic background. In decades, efforts have been made to identify genes associated with the RA development, and human leukocyte antigen (HLA) region is first main

Table 2 The frequency distribution for the $\mathrm{C}+800 \mathrm{~T}$ polymorphism of the BTLA gene in RA patients and normal control groups.

\begin{tabular}{|c|c|c|c|c|}
\hline & $\begin{array}{l}93 \text { RA patients } \\
(\text { count and } \% \text { ) }\end{array}$ & $\begin{array}{l}294 \text { control individuals } \\
\text { (count and \%) }\end{array}$ & $p$-value & OR and $95 \% \mathrm{CI}$ \\
\hline \multicolumn{5}{|l|}{ Genotype frequency } \\
\hline $\mathrm{C} / \mathrm{C}$ & $11(12 \%)$ & $19(6.5 \%)$ & ${ }^{*} p=0.022$ & \\
\hline $\mathrm{C} / \mathrm{T}$ & $30(32 \%)$ & $139(47.3 \%)$ & & \\
\hline $\mathrm{T} / \mathrm{T}$ & $52(56 \%)$ & $136(46.3 \%)$ & & \\
\hline \multicolumn{5}{|l|}{ Homozygous genotype } \\
\hline (C/C or $\mathrm{T} / \mathrm{T}$ genotype) & $63(68 \%)$ & $155(52.7 \%)$ & $p=0.015$ & $1.88(1.12 \sim 3.17)$ \\
\hline \multicolumn{5}{|l|}{ Heterozygous genotype } \\
\hline (C/T genotype) & $30(32 \%)$ & $139(48 \%)$ & & \\
\hline \multicolumn{5}{|l|}{ Allele frequency } \\
\hline $\mathrm{C}$ allele & $52(28 \%)$ & $177(30.1 \%)$ & $p=0.64$ & \\
\hline $\mathrm{T}$ allele & $134(72 \%)$ & $411(69.9 \%)$ & & \\
\hline \multicolumn{5}{|l|}{ Allele carriage frequency } \\
\hline $\mathrm{C}$ allele carriage & $41(44 \%)$ & $158(53.7 \%)$ & $p=0.13$ & \\
\hline $\mathrm{T}$ allele carriage & $82(88 \%)$ & $275(93.5 \%)$ & $p=0.14$ & \\
\hline
\end{tabular}

* $p$-value of the Chi-square test for genotype distribution. 
genetic factor shown to be associated with RA, confirmed by recent several genome scan studies $[19,20]$. However, the HLA appears to only partly influence the RA susceptibility [21]. To further identify non-HLA genes associated with the RA susceptibility, the genetic association method with the candidate gene approach has been popularly used in many studies by using SNPs in candidate genes. Thus, several studies have showed the association of RA susceptibility with certain genes, including TNFR, PADI4, SLC22A4, RUNX1, FCRL3, MHC2TA and PTPN22, although the statistical significance for the association appears to vary in different study populations, except that the RA susceptibility has reproducibly been shown in several populations to be associated with PTPN22 gene, a gene encoding a negative regulator of $\mathrm{T}$ cell activation (reviewed in references 22 and 23).

Since immuno-inhibitory receptors, CTLA-4, PD-1 and BTLA-4, can regulate lymphocyte activation, these receptors become ideal candidate genes in genetic case-control association studies for testing their association with the RA susceptibility. Among these immuno-inhibitory receptors, CTLA-4 gene has been tested in several studies for its association with the RA susceptibility, and the results from different study populations showed the discrepancy [24]. Recently, the PD-1 gene has also been shown to be associated with RA susceptibility in different populations [25, 26]. In this report, we provided the first evidence that the $\mathrm{C}+800 \mathrm{~T}$ SNP in a newly identified immuno-inhibitory receptor, BTLA, is associated with the RA susceptibility in the Taiwanese population. These results together with the association of PTPN22 gene with RA susceptibility lead us to speculate the possibility that the development of RA is governed by the negative immune regulators of lymphocyte activation.

The molecular mechanism that accounts for the association of the $\mathrm{C}+800 \mathrm{~T}$ SNP in the BTLA gene with the RA susceptibility remains to be clarified. Since the genetic variation in the $\mathrm{C}+800$ T SNP results in the amino acid substitution from leucine to proline in the cytoplasmic domain of the BTLA protein, it is possible that two BTLA variants have different structure and/ or function. Thus, one possible explanation for the association of the homozygous $\mathrm{C}+800 \mathrm{~T}$ SNP genotype with the RA susceptibility is that the co-expression of two BTLA variants in the same cell causes the dysregulation in the BTLA signal transduction, since the amino acid substitution for the C+800T SNP is located near the proximal ITIM motif, which has been shown to be required for the association of BTLA and SHP-1 and SHP-2 [27]. Another possible explanation is that the co-expression of two BTLA variants have influenced the tyrosine phosphorylation status, which in turn affects the surface expression level of BTLA, since both BTLA and CTLA-4 are up-regulated after lymphocyte activation and the phosphorylation status of CTLA4 has been shown to influence the surface expression of CTLA-4 [28, 29]. The third possible explanation for the association of the homozygous $\mathrm{C}+800 \mathrm{~T}$ SNP genotype with the RA susceptibility is that two BTLA variants may oligomerize to interact with BTLA, and hetero-oligomer and homo-oligomer may have different HVEM-binding affinity and differentially mediate the BTLA signaling strength and/ or the function of the LIGHT/LT $\alpha$ pathway, because HVEM also can interact with LIGHT and $\mathrm{LT} \alpha$ for co-stimulating $\mathrm{T}$ cell activation [30, 31], and the LIGHT/LT $\alpha$ pathway has also been shown to influence the arthritis occurrence in an animal model [32]. However, it is also possible that the significant association between $\mathrm{C}+800 \mathrm{~T}$ SNP and the RA susceptibility is due to the linkage disequilibrium of the C+800T SNP with other SNPs in the BTLA gene that affect the expression and function of BTLA in lymphocytes.

In conclusion, the data in this study have shown the association of a BLTA SNP with the RA susceptibility. Further study may be needed to investigate the functions of BTLA and this polymorphism in order to have a better insight into the possible involvement of BTLA in the pathogenesis of RA. In addition, it remains to be seen if this SNP is associated with RA in different populations.

\section{Acknowledgements}

I thank Dr Chung-Jen Chen (Chang Gung Memorial Hospital at Kaohsiung, Taiwan) and Yanfeng Lu for review of the manuscript. This 
project is partly supported by a grant from the National Science Council of Taiwan (Grant\# 922314-B-281-002-).

\section{References}

1. Riley J.L. and June C.H., The CD28 family: a T-cell rheostat for therapeutic control of T-cell activation. Blood 105: 13-21, 2005.

2. Greenwald R.J., Freeman G.J. and Sharpe A.H., The B7 Family Revisited. Annu. Rev. Immunol. 23: 515-548, 2005.

3. Nishimura H., Minato N., Nakano T. and Honjo T., Immunological studies on PD-1 deficient mice: implication of PD-1 as a negative regulator for B cell responses. Int. Immunol. 10: 1563-1572, 1998.

4. Watanabe N., Gavrieli M., Sedy J.R., Yang J., Fallarino F., Loftin S.K., Hurchla M.A., Zimmerman N., Sim J., Zang X., Murphy T.L., Russell J.H., Allison J.P. and Murphy K.M., BTLA is a lymphocyte inhibitory receptor with similarities to CTLA-4 and PD-1. Nat. Immunol. 4: 670-679, 2003.

5. Han P., Goularte O.D., Rufner K., Wilkinson B. and Kaye J., An inhibitory Ig superfamily protein expressed by lymphocytes and APCs is also an early marker of thymocyte positive selection. J. Immunol. 172: 59315939, 2004.

6. Shahinian A., Pfeffer K., Lee K.P., Kundig T.M., Kishihara K., Wakeham A., Kawai K., Ohashi P.S., Thompson C.B. and Mak T.W., Differential T cell costimulatory requirements in CD28-deficient mice. Science 261: 609-612, 1993.

7. Dong C., Juedes A.E., Temann U.A., Shresta S., Allison J.P., Ruddle N.H. and Flavell R.A., ICOS co-stimulatory receptor is essential for $\mathrm{T}$-cell activation and function. Nature 409: 97-101, 2001.

8. Tivol E.A., Borriello F., Schweitzer A.N., Lynch W.P., Bluestone J.A. and Sharpe A.H., Loss of CTLA-4 leads to massive lymphoproliferation and fatal multiorgan tissue destruction, revealing a critical negative regulatory role of CTLA-4. Immunity 3: 541-547, 1995.

9. Nishimura H., Nose M., Hiai H., Minato N. and Honjo T., Development of lupus-like autoimmune diseases by disruption of the PD-1 gene encoding an ITIM motif-carrying immunoreceptor. Immunity 11: 141-151, 1999.

10. Nishimura H., Okazaki T., Tanaka Y., Nakatani K., Hara M., Matsumori A., Sasayama S., Mizoguchi A., Hiai H., Minato N. and Honjo T., Autoimmune dilated cardiomyopathy in PD-1 receptor-deficient mice. Science 291: 319322, 2001.

11. Sedy J.R., Gavrieli M., Potter K.G., Hurchla M.A., Lindsley R.C., Hildner K., Scheu S., Pfeffer K., Ware C.F., Murphy T.L. and Murphy K.M., B and T lymphocyte attenuator regulates $\mathrm{T}$ cell activation through interaction with herpesvirus entry mediator. Nat. Immunol. 6: 90 98, 2005.

12. Gonzalez L.C., Loyet K.M., Calemine-Fenaux J., Chauhan V., Wranik B., Ouyang W. and Eaton D.L., A coreceptor interaction between the CD28 and TNF receptor family members B and T lymphocyte attenuator and herpesvirus entry mediator. Proc. Natl. Acad. Sci. USA 102: 1116 $1121,2005$.
13. Wang Y., Subudhi S.K., Anders R.A., Lo J., Sun Y., Blink S., Wang Y., Wang J., Liu X., Mink K., Degrandi D., Pfeffer K. and Fu Y.X., The role of herpesvirus entry mediator as a negative regulator of $\mathrm{T}$ cell-mediated responses. J. Clin. Invest. 115: 711-717, 2005.

14. Kwon B.S., Tan K.B., Ni J., Oh K.O., Lee Z.H., Kim K.K., Kim Y.J., Wang S., Gentz R., Yu G.L., Harrop J., Lyn S.D., Silverman C., Porter T.G., Truneh A. and Young P.R., A newly identified member of the tumor necrosis factor receptor superfamily with a wide tissue distribution and involvement in lymphocyte activation. J. Biol. Chem. 272: 14272-14276, 1997.

15. Miltenburg A.M., van Laar J.M., de Kuiper R., Daha M.R. and Breedveld F.C., T cells cloned from human rheumatoid synovial membrane functionally represent the Th1 subset. Scand. J. Immunol. 35: 603-610, 1992.

16. Quayle A.J., Chomarat P., Miossec P., Kjeldsen-Kragh J., Forre O. and Natvig J.B., Rheumatoid inflammatory T-cell clones express mostly Th1 but also Th2 and mixed (Th0like) cytokine patterns. Scand. J. Immunol. 38: 75-82, 1993.

17. Simon A.K., Seipelt E. and Sieper J., Divergent T-cell cytokine patterns in inflammatory arthritis. Proc. Natl. Acad. Sci. USA 91: 8562-8566, 1994.

18. Arnett F.C., Edworthy S.M., Bloch D.A., McShane D.J., Fries J.F., Cooper N.S., Healey L.A., Kaplan S.R., Liang M.H., Luthra H.S., Medsger T.A., Mitchell D.M., Neustadt A.H., Pinals R.S., Schaller J.G., Sharp J.T., Wilder R.L. and Hunder G.G., The American Rheumatism Association 1987 revised criteria for the classification of rheumatoid arthritis. Arthritis Rheum. 31: 315-324, 1988.

19. Stastny P., Association of the B-cell alloantigen DRw4 with rheumatoid arthritis. N. Engl. J. Med. 298: 869-871, 1978.

20. Choi S.J., Rho Y.H., Ji J.D., Song G.G. and Lee Y.H., Genome scan meta-analysis of rheumatoid arthritis. Rheumatology (Oxford) 45: 166-170, 2006.

21. Seldin M.F., Amos C.I., Ward R. and Gregersen P.K., The genetics revolution and the assault on rheumatoid arthritis. Arthritis Rheum. 42: 1071-1079, 1999.

22. Oliver J.E., Worthington J. and Silman A.J., Genetic epidemiology of rheumatoid arthritis. Curr. Opin. Rheumatol. 18: 141-146, 2006.

23. Dieude P. and Cornelis F., Genetic basis of rheumatoid arthritis. Joint Bone Spine 72: 520-526, 2005.

24. Han S., Li Y., Mao Y. and Xie Y., Meta-analysis of the association of CTLA-4 exon-1 $+49 \mathrm{~A} / \mathrm{G}$ polymorphism with rheumatoid arthritis. Hum. Genet. 118: 123-132, 2005.

25. Prokunina L., Padyukov L., Bennet A., de Faire U., Wiman B., Prince J., Alfredsson L., Klareskog L. and Alarcón-Riquelme M., Association of the PD-1.3A allele of the PDCD1 gene in patients with rheumatoid arthritis negative for rheumatoid factor and the shared epitope. Arthritis Rheum. 50: 1770-1773, 2004.

26. Lin S.-C., Yen J.-H., Tsai J.-J., Tsai W.-C., Ou T.-T., Liu H.-W. and Chen C.-J., Association of a programmed death 1 gene polymorphism with the development of rheumatoid arthritis, but not systemic lupus erythematosus. Arthritis Rheum. 50: 770-775, 2004.

27. Gavrieli M., Watanabe N., Loftin S., Murphy T. and Murphy K., Characterization of phosphotyrosine binding motifs in the cytoplasmic domain of $\mathrm{B}$ and $\mathrm{T}$ lymphocyte attenuator required for association with protein tyrosine phosphatases SHP-1 and SHP-2. Biochem. Biophys. Res. Commun. 312: 1236-1243, 2003. 
28. Shiratori T., Miyatake S., Ohno H., Nakaseko C., Isono K., Bonifacino J.S. and Saito T., Tyrosine phosphorylation controls internalization of CTLA-4 by regulating its interaction with clathrin-associated adaptor complex AP2. Immunity 6: 583-589, 1997.

29. Zhang Y. and Allison J.P., Interaction of CTLA-4 with AP50, a clathrin-coated pit adaptor protein. Proc. Natl. Acad. Sci. USA 94: 9273-9278, 1997.

30. Mauri D.N., Ebner R., Montgomery R.I., Kochel K.D., Cheung T.C., Yu G.L., Ruben S., Murphy M., Eisenberg R.J., Cohen G.H., Spear P.G. and Ware C.F., LIGHT, a new member of the TNF superfamily, and lymphotoxin alpha are ligands for herpesvirus entry mediator. Immunity 8: 21-30, 1998.

31. Watts T.H., TNF/TNFR family members in costimulation of T cell responses. Annu. Rev. Immunol. 23: 23-68, 2005.

32. Fava R.A., Notidis E., Hunt J., Szanya V., Ratcliffe N., Ngam-ek A., de Fougerolles A.R., Sprague A. and Browning J.L., A role for the lymphotoxin/LIGHT axis in the pathogenesis of murine collagen-induced arthritis. J. Immunol. 171: 115-126, 2003. 